\title{
A Comparison Of A Solar Power Satellite Concept To A Concentrating Solar Power System
}

\author{
David V. Smitherman \\ NASA Marshall Space Flight Center, Huntsville, AL, 35812
}

\begin{abstract}
A comparison is made of a Solar Power Satellite concept in geostationary Earth orbit to a Concentrating Solar Power system on the ground to analyze overall efficiencies of each infrastructure from solar radiance at $1 \mathrm{AU}$ to conversion and transmission of electrical energy into the power grid on the Earth's surface. Each system is sized for a 1-gigawatt output to the power grid and then further analyzed to determine primary collector infrastructure areas. Findings indicate that even though the Solar Power Satellite concept has a higher end-to-end efficiency, that the combined space and ground collector infrastructure is still about the same size as a comparable Concentrating Solar Power system on the ground.
\end{abstract}

